\title{
Electric-Field-Assisted Facile Synthesis of Metal Nanoparticles
}

Qi Zhou, ${ }^{\mathrm{a}, *}$ Jingjing Zhang, ${ }^{\mathrm{a}, \mathrm{b}}$ Tony Saba, ${ }^{\mathrm{c}}$ Zhen Yue, ${ }^{\mathrm{a}, \mathrm{b}}$ Wei Li, ${ }^{\mathrm{b}}$ James A. Anderson, ${ }^{\mathrm{c}}$ and Xiaodong Wang,*

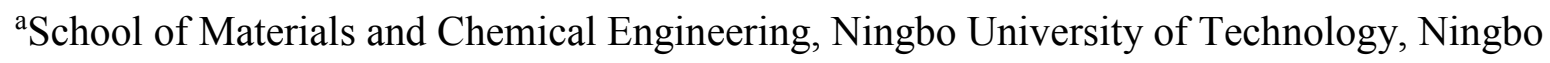
315016, Zhejiang, P.R. China.

${ }^{b}$ Ningbo Key Laboratory of Specialty Polymers, School of Material Science and Chemical Engineering, Ningbo University, Ningbo 315211, Zhejiang, P.R. China.

${ }^{\mathrm{c}}$ Chemical and Materials Engineering, School of Engineering, University of Aberdeen, Aberdeen AB24 3UE, Scotland, United Kingdom.

Corresponding Author:

Qi Zhou, E-mail: zhouqi@nbut.edu.cn

Xiaodong Wang, Email: x.wang@abdn.ac.uk 
ABSTRACT: The use of electric field represents a promising strategy to overcome the existent challenges in synthesizing metal nanoparticles. A facile and clean method for the preparation of water-soluble metal nanoparticles with a narrow particle distribution is reported here based on the electric-field-driven phase inversion procedure. Bioderived chitosan was used to stabilize the metal nanoparticles and formic acid was employed as both a solvent (for the polymer) and a reductant (for the metal). The electric field has been characterized to modify the hydrogen bonds of the chitosan and promote the stabilization and reduction of metal nanoparticles. Taking silver as an example, the nanoparticles obtained lay in the range of 2-6 nm with a mean size of $3.4 \mathrm{~nm}$. The produced chitosan/Ag nanocomposites can be used in homogenous (water-soluble) form for catalysis and heterogeneous form (as a solid film) for antibiosis. Exceptional performance in the selective regeneration of cofactor NADH $(T O F=$ $582 \mathrm{~h}^{-1}$ ) and an improved antibacterial performance were attained. Successful preparation of metallic $\mathrm{Cu}, \mathrm{Au}$ and Pt nanoparticles $(\sim 1-2 \mathrm{~nm})$ confirms the universality of this method for synthesizing functional nanomaterials with various potential applications. 


\section{Introduction}

Metal nanoparticles (NPs) have been used in a diverse range of fields including energy, environment, catalysis, electronics, sensing, imaging and biomedical sciences, etc. ${ }^{1-3}$ To prepare these metal NPs, (chemical) reduction of metal ions is still one of the most popular steps (relative to cathodic corrosion, ion sputtering, impregnation and incipient wetness methods, and gas-phase synthesis like laser vaporization) owing to the high efficiency in controlling the surface structures and morphology of the NPs. ${ }^{4-7}$ To date, however, the precision during preparation of metal nanoparticles with controllable dimension, distribution and shape remains a challenge, since the high surface energy of the NPs always leads to aggregation. ${ }^{4-10}$ A polymer stabilizing agent is invariably added during the synthesis in order to prevent aggregation of the metal NPs. The use of polymer stabilizers (e.g. polyvinylpyrrolidone, polyethylene glycol and poly(vinyl alcohol)) and highlyreactive/hazardous reducing agent (e.g. sodium borohydride and hydrazine) results in complex and environmentally-unfriendly preparation procedures. ${ }^{11-15}$ In this study, an innovative, facile and clean method for NPs synthesis based on external electric-field-assisted phase inversion with improved properties is reported (e.g. size/distribution and catalytic activity), opening up a new direction in the preparation of nanomaterials.

The control of metal NPs during synthesis largely depends on the polar functionalities of the stabilizers (notably hydrogen bonds) which direct the dynamics of the polymer chains and the interactions between the metal ions and polymer molecules, thus determining the morphology and microstructure of the metal NPs. ${ }^{11-15}$ The approach here is to use chitosan (CS), a natural, non-toxic and antimicrobial polymer $(\beta-1,4-\mathrm{N}$-acetylglucosamine $),{ }^{16-19}$ as an ion capping agent, where its abundant hydrogen bonds and complex hydrogen bonding environment (i.e. intermolecular $-\mathrm{NH}_{2}$ and intramolecular $-\mathrm{OH}$, Scheme 1), after rationalisation, can facilitate the stabilization and manipulation of the growth of metal NPs. As with other stabilizers, $\mathrm{CS}$ is soluble at low $\mathrm{pH}$ via amine pronation. ${ }^{16-18}$ Formic acid is 
proposed as a multifunctional solvent that can also act as a reducing agent for metal ions and moreover, upon redox reaction, generate water-soluble CS (via the formation of ionic bonds between the $\mathrm{CO}_{2}$ produced and the $-\mathrm{NH}_{2}$ groups). The water-soluble feature is of particular significance as metals encapsulated in a matrix/cluster have shown wide spread applications in the chemical sciences. ${ }^{3,6,10,20-24}$ However, the reported synthesis of CS/metal NPs in the literature produced only insoluble materials. ${ }^{22-24}$ The proposal here can be employed successfully with the assistance of electric field during the solution casting, where the dipoles of the polar functionalities in the CS chains can be controlled to depress the formation of hydrogen bonds and serve to $(i)$ stabilize the dispersion of metal NPs and (ii) improve the interfacial interaction/chelation between the precursors and the CS molecules, thus promoting the reductive efficiency of metal ions. This hypothesis is based on our recent findings in applying electrically assisted phase inversion to control the dipole orientation of the polar functionalities in the polyamide 6 where the presence of the electric field greatly influenced the alignment of hydrogen bonding and crystallinity of the polymers. ${ }^{25,26}$

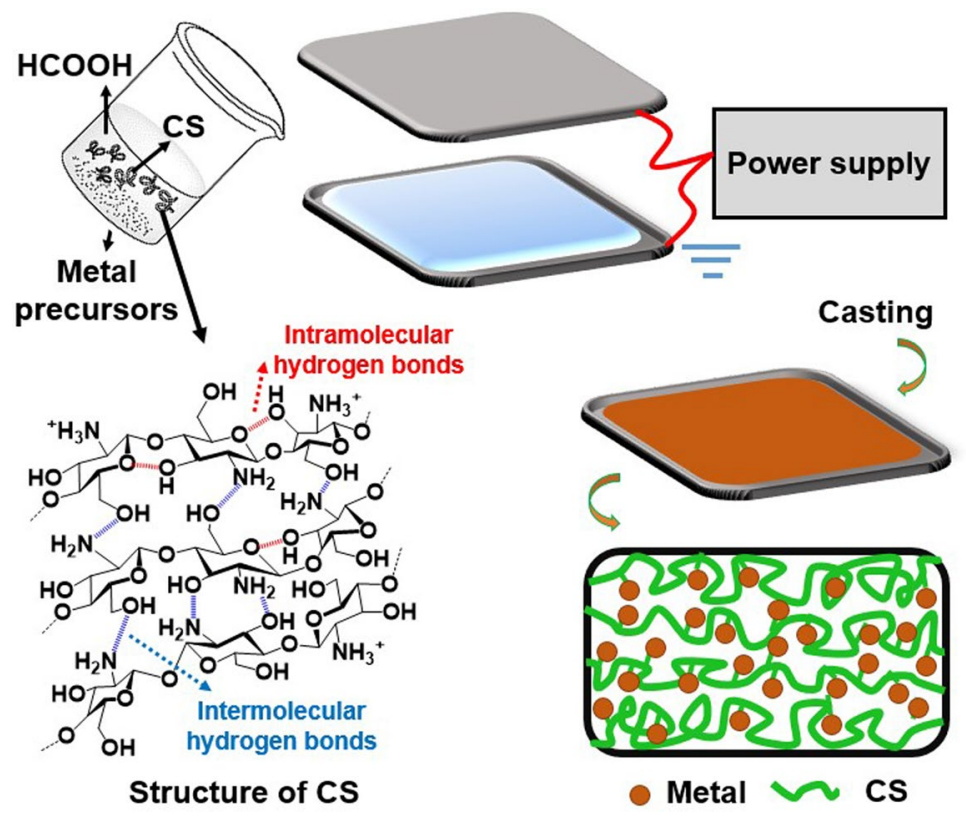

Scheme 1. Schematic process of electric-field-assisted synthesis of chitosan/metal NPs.

This is the first report on electric-field-assisted method for confined metallic $(\mathrm{Ag}, \mathrm{Cu}, \mathrm{Au}$, and Pt) NPs synthesis (Scheme 1), which has proven effective in producing well-dispersed 
and narrow-particle size distributed metallic NPs. The prepared metal NPs are firmly confined in the CS matrix and can exist and be used in water-soluble homogeneous form or as a heterogeneous film. Taking Ag as an example, we report here the performance of the homogeneous Ag NPs in the selective regeneration of nicotinamide adenine dinucleotide $(\mathrm{NADH})$ and the antibacterial property of the heterogeneous Ag NPs (CS/Ag nanocomposites) as selected applications.

\section{Materials and Methods}

2.1 Materials. Chitosan (powder, $85.0 \%$ of the degree of deacetylation and $800 \mathrm{mPa} \cdot \mathrm{s}$ of viscosity) was purchased from Sinopharm Chemical Reagent Company. $\mathrm{AgNO}_{3}$ (99.8\%), $\mathrm{Cu}\left(\mathrm{NO}_{3}\right)_{2}(99 \%), \mathrm{HAuCl}_{4}(99.8 \%)$ and $\mathrm{H}_{2} \mathrm{PtCl}_{6}(99 \%)$ were obtained from Aladdin. Formic acid $(\mathrm{HCOOH}, 99 \%)$ was purchased from J\&K Scientific. $\beta$-Nicotinamide adenine dinucleotide hydrate $\left(\mathrm{NAD}^{+}, \geq 96.5 \%\right), \beta$-nicotinamide adenine dinucleotide reduced disodium salt hydrate $(\mathrm{NADH}, \geq 94 \%), \mathrm{KH}_{2} \mathrm{PO}_{4}(\geq 99 \%)$ and $\mathrm{K}_{2} \mathrm{HPO}_{4}(\geq 98 \%)$ were obtained from Sigma-Aldrich. The enzymatic assay (EnzyChrom NAD ${ }^{+}$NADH Assay Kit, E2ND-100) was supplied by Universal Biologicals. All the chemicals were used as received without further purification.

2.2 Preparation of CS/Ag nanocomposites. $200 \mathrm{mg}$ of CS was added into $20 \mathrm{ml}$ of formic acid and was stirred at room temperature for $12 \mathrm{~h}$ to achieve a homogeneous solution. $12 \mathrm{mg}$ of $\mathrm{AgNO}_{3}$ was added into the $\mathrm{CS} /$ formic acid and was stirred for another $24 \mathrm{~h}$ to make the $\mathrm{CS} / \mathrm{AgNO}_{3}$ solution with light yellowish color. The experimental equipment was shown in our previous work. ${ }^{1,2}$ Two metal sheets with polyformaldehyde coating were used as electrode plates. At room temperature and controlled moisture $(65 \%)$, the polymer solution $(2 \mathrm{ml})$ was cast/spread onto the bottom plate (with an area of $56 \mathrm{~cm}^{2}$ ) which was connected to the ground. The distance of the metal sheets was set at $8 \mathrm{~mm}$, and the electric field voltage was set at 0,1 , $3,5,7 \mathrm{kV}$ by a high voltage d.c. power source, respectively. Thus the samples prepared were named as CS/Ag-0, CS/Ag-1, CS/Ag-3, CS/Ag-5, and CS/Ag-7, respectively. After the formic 
acid were evaporated, the yellowish CS/Ag films with an average thickness of $40 \pm 2 \mu \mathrm{m}$ was achieved. The membrane was dried under vacuum at $60{ }^{\circ} \mathrm{C}$ for $24 \mathrm{~h}$. For comparison purposes, pure CS membrane was synthesized according to the same procedure without adding $\mathrm{AgNO}_{3}$. For synthesizing $\mathrm{CS} / \mathrm{Cu}, \mathrm{CS} / \mathrm{Au}$ and $\mathrm{CS} / \mathrm{Pt}$ nanocomposites, $12 \mathrm{mg}$ of $\mathrm{Cu}\left(\mathrm{NO}_{3}\right)_{2}, \mathrm{HAuCl}_{4}$ and $\mathrm{H}_{2} \mathrm{PtCl}_{6}$ were used instead of $\mathrm{AgNO}_{3}$, respectively. In order to further reduce $\mathrm{Pt}^{4+}, 2 \mathrm{mg}$ of $\mathrm{H}_{2} \mathrm{PtCl}_{6}$ was also used. The weight percentage used in the text is defined by the mass of metal precursor over the mass of CS used, i.e. $12 \mathrm{mg} / 200 \mathrm{mg}=6 \mathrm{wt} \%, 2 \mathrm{mg} / 200 \mathrm{mg}=1 \mathrm{wt} \%$.

2.3 Characterization. To study the morphology of the polymer surface, scanning electron microscopy (SEM) was conducted over a HITACHI S-4800 SEM using an acceleration voltage of $10 \mathrm{kV}$. Samples were coated by $\mathrm{Pt}$ before the observation. Attenuated total reflectance infrared resonance (ATR) analysis of the obtained film samples was measured using an IR spectroscopy (Thermo Nexus 670 FTIR ESP) at a resolution of $2 \mathrm{~cm}^{-1}$ with a range of $750-4000 \mathrm{~cm}^{-1} .64$ scans were collected for each spectrum. The samples were dried at $60{ }^{\circ} \mathrm{C}$ under the vacuum for 2 days prior to the measurement. X-ray diffraction (XRD) measurements were carried out on a Bruker GADDS diffract meter with $\mathrm{Cu} \mathrm{K} \alpha$ radiation at $40 \mathrm{kV}$ and $40 \mathrm{~mA}(\lambda=0.154 \mathrm{~nm})$. The step size was $0.02^{\circ}$ and test range was from $5^{\circ}$ to $70^{\circ}$.

The morphology of metal NPs in the CS matrix were observed by a transmission electron microscope (TEM, JEM-1230, Japan). Samples were cut by a freezing microtome before analysis by the TEM. Software Image $\mathrm{J}$ was used to calculate the average size and distribution of metal NPs based on up to 250 NPs from the TEM images. The elemental analysis of CS and CS/metal films were studied by X-ray photoelectron spectroscopy (XPS) and performed with Escalab 250Xi using Al $\mathrm{K} \alpha$ radiation as the $\mathrm{X}$-ray source $(300 \mathrm{~W}, 1486.3 \mathrm{eV})$. The vacuum chamber was about $5 \times 10^{-9}$ Torr. Auger electron spectroscopy (XAES) was carried out on a PHI 1600 ESCA instrument (PE Company) equipped with an Al K $\alpha$ X-ray radiation source $(h v=1486.6 \mathrm{eV})$. The calibration of binding energy of the spectra was executed on the basis of the $\mathrm{C} 1 \mathrm{~s}$ peak of carbon at $284.6 \mathrm{eV}$. 
2.4 Solubility of the synthesized CS/Ag nanocomposites. $0.02 \mathrm{~g}$ of synthesized CS/Ag nanocomposites and CS films were solved into $10 \mathrm{ml}$ of distilled water for $24 \mathrm{~h}$. The solution was measured by a UV-vis spectra (TU-1901 spectrophotometer, PERSEE Corp, China) in the range of 200 to $800 \mathrm{~nm}$. The solution was mixed with dried $\mathrm{KBr}$ and then was pressed into a film. This membrane was dried for 10 mins to remove the residual water and then analyzed by the infrared spectra. The film was measured with a 32 of scans and a $4 \mathrm{~cm}^{-1}$ of resolution.

2.5 Catalytic activity. $\mathrm{NAD}^{+} \rightarrow \mathrm{NADH}$ regeneration over the water-soluble Ag NPs was carried out in a $\operatorname{Parr}^{\circledR} 5500$ compact reactor (with a $\operatorname{Parr}^{\circledR} 4848$ reactor controller) at $37^{\circ} \mathrm{C}, \mathrm{pH}$ $=7.0$ and $10 \mathrm{~atm}$ of $\left(\mathrm{H}_{2}\right)$ pressure. $\mathrm{CS} / \mathrm{Ag}$ was dissolved first under mild agitation for $3 \mathrm{~h}$. In a typical experiment, phosphate buffered solution $\left(50 \mathrm{~cm}^{3} 0.1 \mathrm{M}\right)$ containing $\mathrm{NAD}^{+}(3.0 \mathrm{mM})$ were loaded in the reactor $\left(\mathrm{NAD}^{+} / \mathrm{Ag}\right.$ molar ratio $\left.=3000\right)$. The system was flushed (three times) with $\mathrm{N}_{2}$ and the temperature was allowed to stabilize. Hydrogen gas was then introduced, the system was pressurized and stirring (at $1500 \mathrm{rpm}$ ) engaged (time $t=0$ for reaction). A non-invasive liquid sampling system via syringe/in-line filters allowed a controlled removal of aliquots from the reactor. Catalytic regeneration is quantified in terms of initial rates obtained from a linear regression of temporal NADH concentration profiles ${ }^{4}$ with initial TOF calculated using Ag dispersion obtained from TEM analysis taking into account the degree of $\mathrm{Ag}$ reduction in different catalysts. The NADH selectivity was established by a commercially available independent enzymatic assay (EnzyChrom $\mathrm{NAD}^{+} / \mathrm{NADH}$ Assay Kit, E2ND-100), as reported in detail by Li et al. ${ }^{5}$ Replicated reactions in both catalytic systems delivered raw data reproducibility to within $\pm 7 \%$.

2.6 Bactericidal activity. The antibacterial test over the heterogeneous Ag NPs (CS/Ag nanocomposities film) was executed according to similar approaches reported in the literature. ${ }^{6-9}$ The ATCC6538 staphylococcus aureus (S. aureus) was used as a model bacteria. The required nutrient agar media for $S$. aureus was prepared from tryptic soy agar. Sterilization of the agar media was done at a pressure of $103.4 \times 10^{3} \mathrm{~Pa}$ for $15 \mathrm{~min}$ at $121{ }^{\circ} \mathrm{C}$. 
The respective sterilized media were then transferred into sterilized petri dishes on a clean bench. After solidification of the growth media, bacterial cultures $(50 \mu \mathrm{L})$ were inoculated by uniformly streaking the solidified agar surface. The bacterial cultures were incubated for $8 \mathrm{~h}$ at $37^{\circ} \mathrm{C}$ in an incubation chamber. Finally, the developed inhibition zones were photographed.

\section{Results and discussion}

The proposed electric-field-assisted method was firstly examined by the preparation and characterization of Ag NPs as a representative case. All the synthesized CS and CS/Ag films present a non-porous structure. The pure CS membrane looks colorless, while the CS/Ag films varied from yellowish to brownish in color owing to the reduction and distribution of Ag NPs, consistent with the literature. ${ }^{27}$ The attenuated total reflectance infrared (ATR) spectra of the CS/Ag nanocomposites are shown in Figure 1 and provide characteristics of the bonding environment. For the pure CS film (Figure 1a), the band around $3360 \mathrm{~cm}^{-1}$ can be ascribed to the $\mathrm{O}-\mathrm{H}$ and $\mathrm{N}-\mathrm{H}$ stretching modes. The peaks at 2920 and $2852 \mathrm{~cm}^{-1}$ correspondent to the $\mathrm{CH}_{3}$ stretching vibrations of the pyranose ring of $\mathrm{CS}$. The $\mathrm{C}=\mathrm{O}$ group, generated by the residual acetyl groups of the CS, is evident at $1720 \mathrm{~cm}^{-1}$. The peak at 1660 $\mathrm{cm}^{-1}$ is attributable to the $\mathrm{N}-\mathrm{H}$ bending mode (amide I band, C-O stretch of acetyl group) while the peak at $1580 \mathrm{~cm}^{-1}$ results from the N-H stretching mode (amide II). The bending mode of the $\mathrm{OH}$ groups is detected at $1380 \mathrm{~cm}^{-1}$ whereas the band at $1326 \mathrm{~cm}^{-1}$ corresponds to $\mathrm{CH}_{2}$ wagging vibrations. The anti-symmetrical stretching and symmetric stretching of C-O-C bridge are shown at 1154 and $1073 \mathrm{~cm}^{-1}$, respectively. ${ }^{27-29}$ In contrast to the pure CS, the bands at 3360, 1580 and $1660 \mathrm{~cm}^{-1}$ of $\mathrm{CS} / \mathrm{Ag}-0$, decrease (in terms of intensity) and shift slightly to lower wavenumbers, showing the interaction ${ }^{27-29}$ of primary amino groups of the CS with silver (see Figure 1a, c). Interestingly, it is evident from the ATR spectra of all the CS/Ag samples that the intensities at $1660 \mathrm{~cm}^{-1}$ and $1720 \mathrm{~cm}^{-1}$ increase gradually with an 
increase in electric field voltage (see Figure 1b, d). These results indicate that more $\mathrm{C}=\mathrm{O}$ groups are generated with increasing electric field due to the participation of formic acid in the reduction of $\mathrm{Ag}$ ions, i.e. high electric field strength promotes the efficiency of $\mathrm{Ag}$ reduction. In addition, the intensities of features at 3330 and $1380 \mathrm{~cm}^{-1}$ of the $\mathrm{CS} / \mathrm{Ag}$ samples increase initially with increasing electric field voltage (to $1 \mathrm{kV}$ ) and then decrease with a further increase (to $7 \mathrm{kV}$ ). This observation suggests that the electric field strength can control the hydrogen bonding environment where a gradual relaxation of the hydrogen bonds can be achieved by increasing the electric field strength (from 1 to $7 \mathrm{kV}$ ). ${ }^{25-29}$ The dependence of hydrogen bonding on the electric field strength can be linked to the orientation of the dipoles of the polar groups (i.e. $\mathrm{N}-\mathrm{H}, \mathrm{C}-\mathrm{O}, \mathrm{O}-\mathrm{H})$ in the electric field direction, which rearranges the conformation of CS chains and results in decreased intensities at 2920 and $2852 \mathrm{~cm}^{-1} .25,26,30,31$
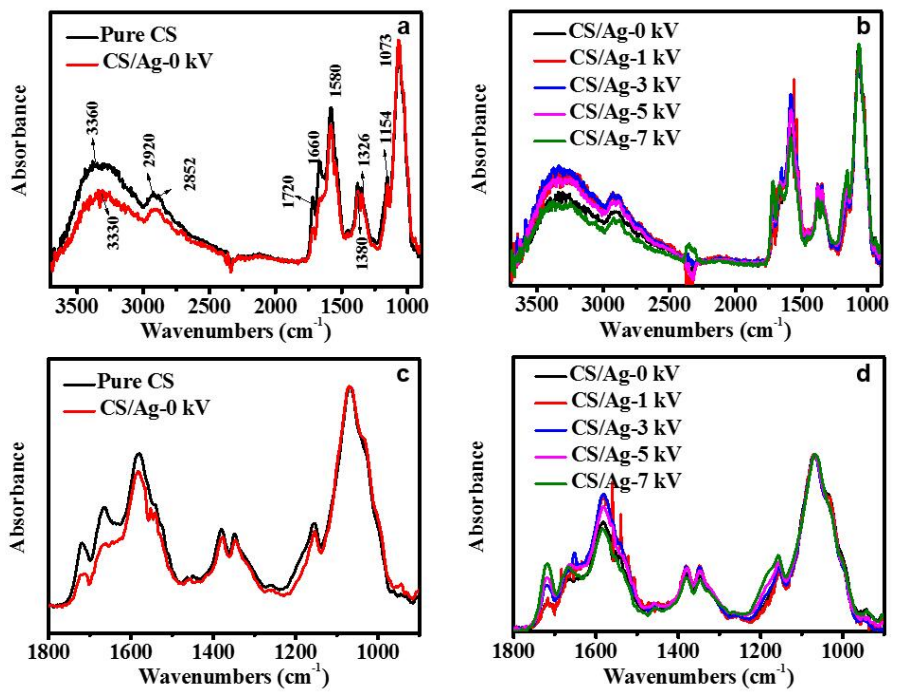

Figure 1. ATR spectra of CS and CS/Ag nanocomposites, a, b) the full ATR spectra, c, d) the enlarged spectra of a and b, ranging from 1800 to $750 \mathrm{~cm}^{-1}$. Pure CS was synthesized in the absence of electric field. 


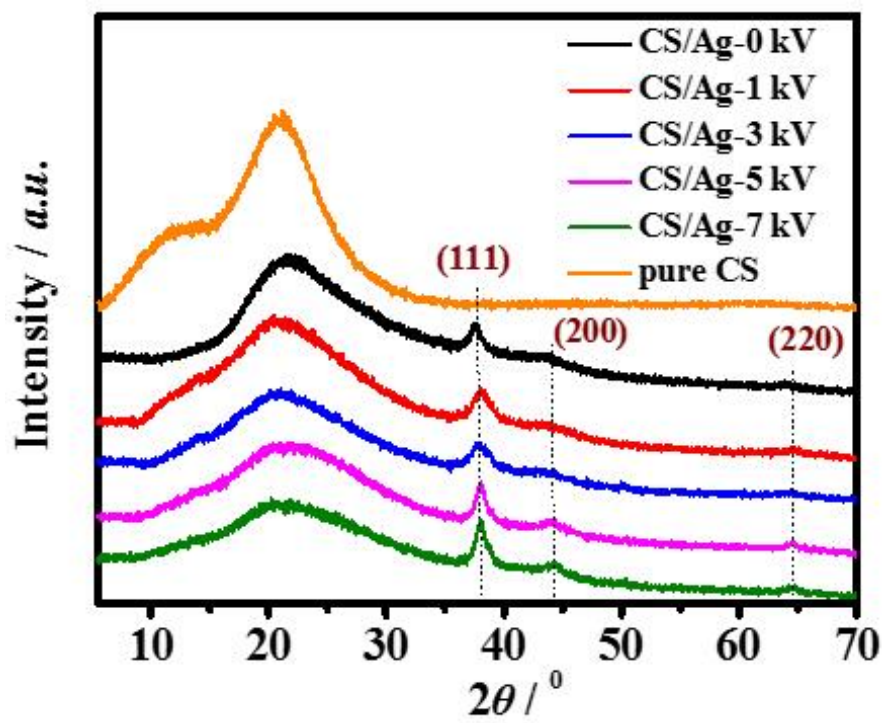

Figure 2. XRD patterns of CS and CS/Ag nanocomposites synthesized at different voltages.

It is worth noting that both the intramolecular hydrogen bonds (between the ring $\mathrm{O} 5$ and hydroxyl O3 atoms, provide rigidity to a single chitosan chain, see Scheme 1) and the intermolecular hydrogen bonds (between the amine $\mathrm{N}$ and hydroxyl $\mathrm{O} 3$ as well as $\mathrm{O} 6$ atoms, corresponding to the self-assembly and the antiparallel packing of the chains in the crystals) contribute to stabilization of metal ions. ${ }^{16-19}$ The X-ray powder diffraction (XRD) patterns of $\mathrm{CS}$ and $\mathrm{CS} / \mathrm{Ag}$ nanocomposites synthesized at different voltages are given in Figure 2 and distinguish the variation of the two types of hydrogen bonds upon electric field. The pure CS shows two reflection peaks at $\sim 12^{\circ}$ and $\sim 20^{\circ}$, which can be ascribed to the representations of intermolecular and of intramolecular hydrogen bonds, respectively. ${ }^{16-19}$ The relative intensities of the two peaks are consistent with the fact that there are more intramolecular than intermolecular hydrogen bonds in the CS polymer and the bond strength of the former is stronger than that of the latter. ${ }^{16,18,19}$ The peak at $\sim 12^{\circ}$ almost disappears in the CS/Ag-0 sample, indicating the loss of intermolecular hydrogen bonds. This may be due to the strong charge interaction between protonated amino groups and silver ions, which hinders the formation of intermolecular hydrogen bonds. ${ }^{16-19,28,31}$ However, the same peak reappears under electric field $(1 \mathrm{kV})$ and gradually decreases with further increment of electric field (see Figure 2), suggesting that the intermolecular hydrogen bonding was first enhanced and then 
weakened by the electric field. Furthermore, the diffraction peak at around $20^{\circ}$ exhibited the same trend, i.e. enhanced at $1 \mathrm{kV}$ and then became broader at higher electric strength, showing the inhibition of intramolecular hydrogen bonding. ${ }^{16-19}$ These results are in very good agreement with that of the ATR spectra discussed previously (see Figure 1b, d). The enhanced hydrogen bonding (i.e. at $1 \mathrm{kV}$ ) may arise as more silver ions are reduced to $\mathrm{Ag}^{0}$ (relative to $0 \mathrm{kV}$ ) blocking the charge interaction and thus allowing re-formation of the intermolecular hydrogen bonds. ${ }^{16,31}$ Further decreased hydrogen bonding can arise due to the enhanced dipole orientation of the polar functionalities in the CS chains. ${ }^{25,26,30,31}$ Meanwhile, the three diffraction peaks at $38^{\circ}, 44^{\circ}$ and $64^{\circ}$, corresponding to the $\mathrm{Ag}(111),(200)$ and (220) lattice planes, ${ }^{17}$ respectively, confirm the formation of metallic Ag crystals in the CS matrix.

The morphology, particle size and distribution are critical in determining the ultimate properties of the metal NPs. ${ }^{1-10}$ Thus, transmission electron microscopy (TEM) analysis of the CS/Ag films was conducted. The results (Figure 3) indicate the formation of spherical silver nanoparticles (mean sizes $d<9 \mathrm{~nm}$ ) distributed homogeneously through the CS matrix, thereby demonstrating the feasibility of this electric-field-assisted method. The average size and the distribution of Ag NPs first increases and then decreases with an increase in the electric field strength (see Figure 4), consistent with the changes in the hydrogen bonding environment. The smallest size with a uniformed distribution (i.e. $d=3.4 \pm 0.7 \mathrm{~nm}$ ) of Ag NPs is achieved at $7 \mathrm{kV}$. The lattice plane with a spacing of $2.36 \AA$ indicates the formation of $\mathrm{Ag}^{0}$ nanoclusters oriented in the (111) plane (Figure 3f). This is a result of the loss of inter- and intra- molecular hydrogen bonds at high voltage which enhances availability of the amino groups to stabilize the silver ions. ${ }^{16-19}$ To the best of our knowledge, these are the smallest Ag NPs size reported in a CS matrix. 


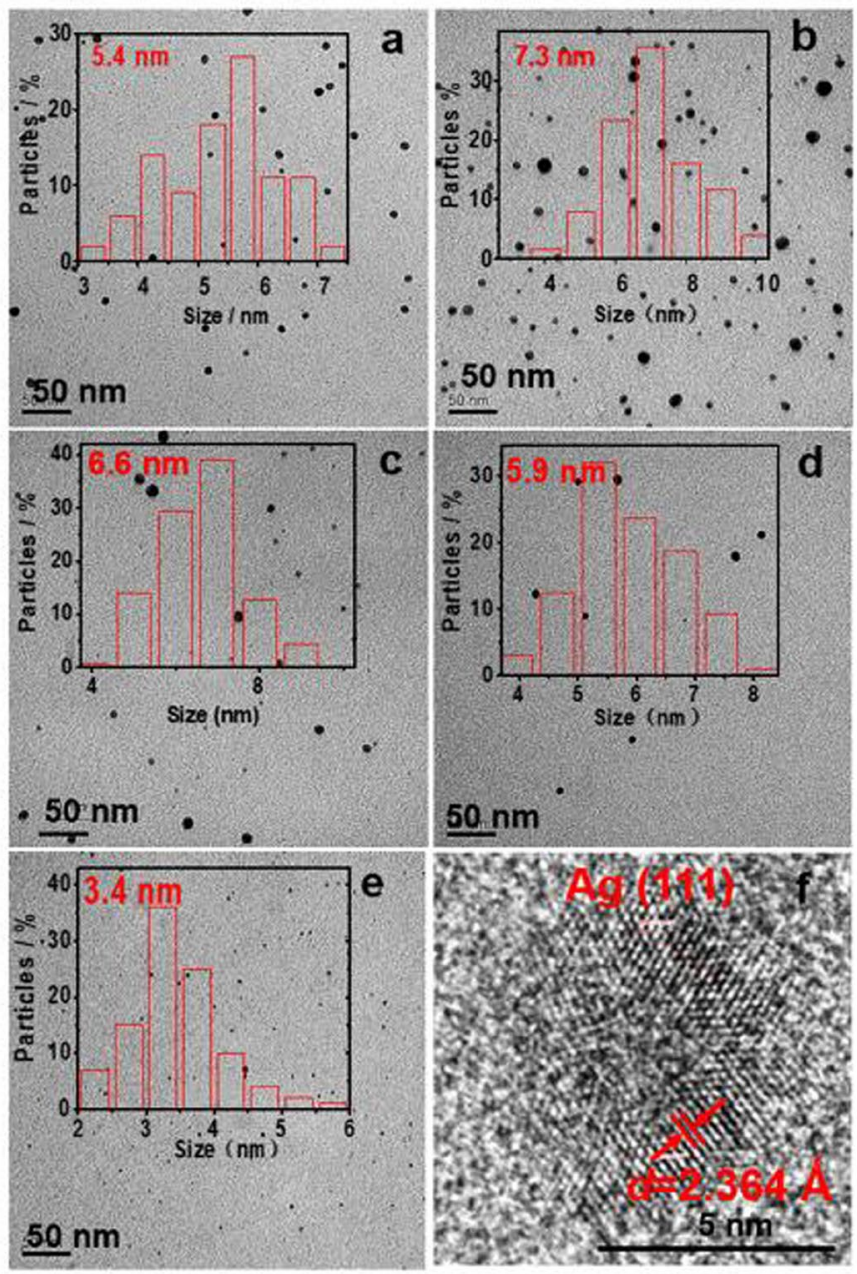

Figure 3. TEM images of CS/Ag nanocomposites under different voltages, a) $0 \mathrm{kV}$, b) $1 \mathrm{kV}$, c) $3 \mathrm{kV}$, d) $5 \mathrm{kV}$, e) $7 \mathrm{kV}$ and f) HRTEM of e.

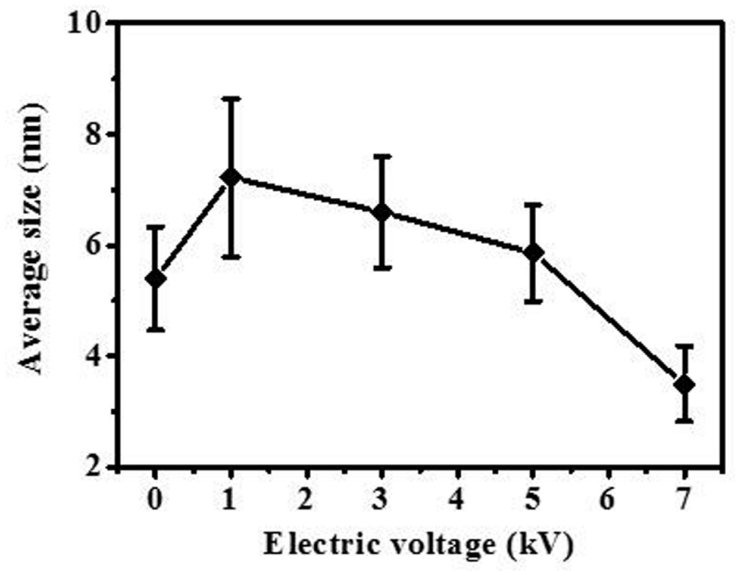

Figure 4. The average size with standard deviation of Ag NPs as a function of electric voltages. 
A typical XPS survey spectrum of CS/Ag nanocomposites is presented in Figure S1 with detailed elemental analysis of Ag shown in Figure 5. Two characteristic peaks of Ag were observed and can be attributed to the $\operatorname{Ag} 3 d_{3 / 2}(\sim 374.0 \mathrm{eV})$ and $\operatorname{Ag} 3 d_{5 / 2}(\sim 368.0 \mathrm{eV}) .^{32}$ This doublet can be resolved into two components, where the lower binding energy feature (i.e. 373.0 and $367.2 \mathrm{eV}$, see Figure 5a) indicates the presence of $\mathrm{Ag}$ ions and the higher one represents metallic $\mathrm{Ag}\left(\mathrm{Ag}^{0}\right)$. These results suggest that $73.4 \mathrm{wt} \%$ of the silver is already in metallic state without the application of the electric field. Mady et al. observed that silver ions can be partially reduced (by hydrazine) to metal state by microwave-assisted self-assembly precipitation. ${ }^{32} 96 \mathrm{wt} \%$ of $\mathrm{Ag}$ ions were reduced to $\mathrm{Ag}^{0}$ at an external voltage of $3 \mathrm{kV}$ and all of the $\mathrm{Ag}$ are in the metal state at an external voltage of $7 \mathrm{kV}$ (Figure $5 \mathbf{b}, \mathbf{c}$ ), suggesting effective metal reduction in the absence of strong reducing agents such as sodium borohydride or hydrazine. Upon increasing the electric field, the inter- and intramolecular hydrogen bonds were destroyed, releasing additional $\mathrm{NH}_{2}$ groups ${ }^{16-19}$ that consume more $\mathrm{CO}_{2}$ which promote the redox reaction for $\mathrm{Ag} \mathrm{NPs}$ generation $\left(\mathrm{HCOOH}+2 \mathrm{AgNO}_{3}=2 \mathrm{Ag}+\mathrm{CO}_{2}+2 \mathrm{HNO}_{3}\right){ }^{33}$ The work of Yanson et al. should be highlighted here as they reported extreme cathodic corrosion can also generate metal NPs, although based on a different mechanism, where extreme negative potentials favored metal NPs production. ${ }^{5,7}$
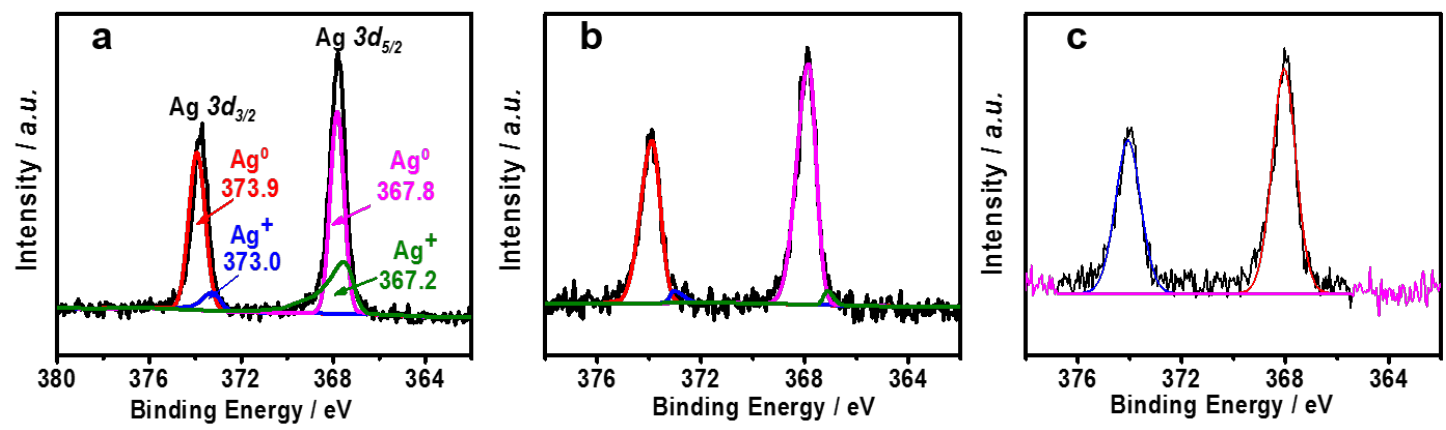

Figure 5. XP spectra for $\mathrm{Ag} 3 d$ region of $\mathrm{CS} / \mathrm{Ag}$ NPs films under a variety of voltages, a) 0 $\mathrm{kV}$, b) $3 \mathrm{kV}$ and c) $7 \mathrm{kV}$. 
It has been demonstrated so far that the electric-field-assisted method can be used to prepare metallic Ag NPs immobilized in the CS matrix as a heterogeneous film, which in turn allow us to consider whether it would be possible to produce water-soluble Ag NPs. It is noteworthy here that there has been no reported attempt in the literature to dissolve CS confined/encapsulated metal NPs. The $\mathrm{O} 1 s, \mathrm{~N} 1 s$ and $\mathrm{C} 1 s$ spectra of the CS/Ag nanocomposites were thus further analyzed by the deconvolution of XPS curves (Figure S2S4). Comparing to the data of the pure CS (see Figure S5), the fractions of O $1 s$ at $531.8 \mathrm{eV}$ (corresponding to the $\mathrm{O}=\mathrm{C}$ ), $\mathrm{N} 1 s$ at $401.5 \mathrm{eV}$ (corresponding to the $-\mathrm{NH}^{3+}$ ), $\mathrm{C} 1 s$ at $286.4 \mathrm{eV}$ (corresponding to the $\mathrm{C}-\mathrm{N}, \mathrm{C}-\mathrm{O}$ or $\mathrm{C}-\mathrm{O}-\mathrm{C}$ ) and at $288.2 \mathrm{eV}$ (corresponding to the $\mathrm{C}=\mathrm{O}$ or $\mathrm{O}$ $\mathrm{C}-\mathrm{O})$, all increase in the $\mathrm{CS} / \mathrm{Ag}$ samples with an increase in electric field strength. These results indicate that the formic acid was involved in the reduction of the silver ions, generating $\mathrm{CO}_{2}$ that then combines/coordinates with the $\mathrm{NH}_{2}$ groups of $\mathrm{CS}$ chains forming $-\mathrm{NH}_{3}{ }^{+} \mathrm{COO}^{-}{ }^{32,34}$ These $-\mathrm{NH}_{3}{ }^{+} \mathrm{COO}^{-}$groups will make the CS chains water-soluble whereby (Figure S6) the CS/Ag nanocomposites can be dissolved in water, creating a transparent/homogeneous yellowish solution. This transparent solution was characterized by UV-vis (Figure S7), in which the typical absorbance of CS is present at $285 \mathrm{~nm}$ and the $\mathrm{Ag}$ NPs give a feature at $400 \mathrm{~nm} .{ }^{35}$ The FTIR spectra of the CS/Ag water solution are also shown in Figure S7, where the absorbances due to CS molecules appeared at 3400, 2930, 2056, 1630, 1368 and $1074 \mathrm{~cm}^{-1} \cdot{ }^{27-29}$ These features suggest that the CS chains are soluble in water while maintaining the metallic state of Ag NPs, making it possible for homogeneous applications (e.g. catalysis). It is worth mentioning that the above homogenous state of the CS/Ag solutions is stable for over two months (i.e. no detection of precipitate). 


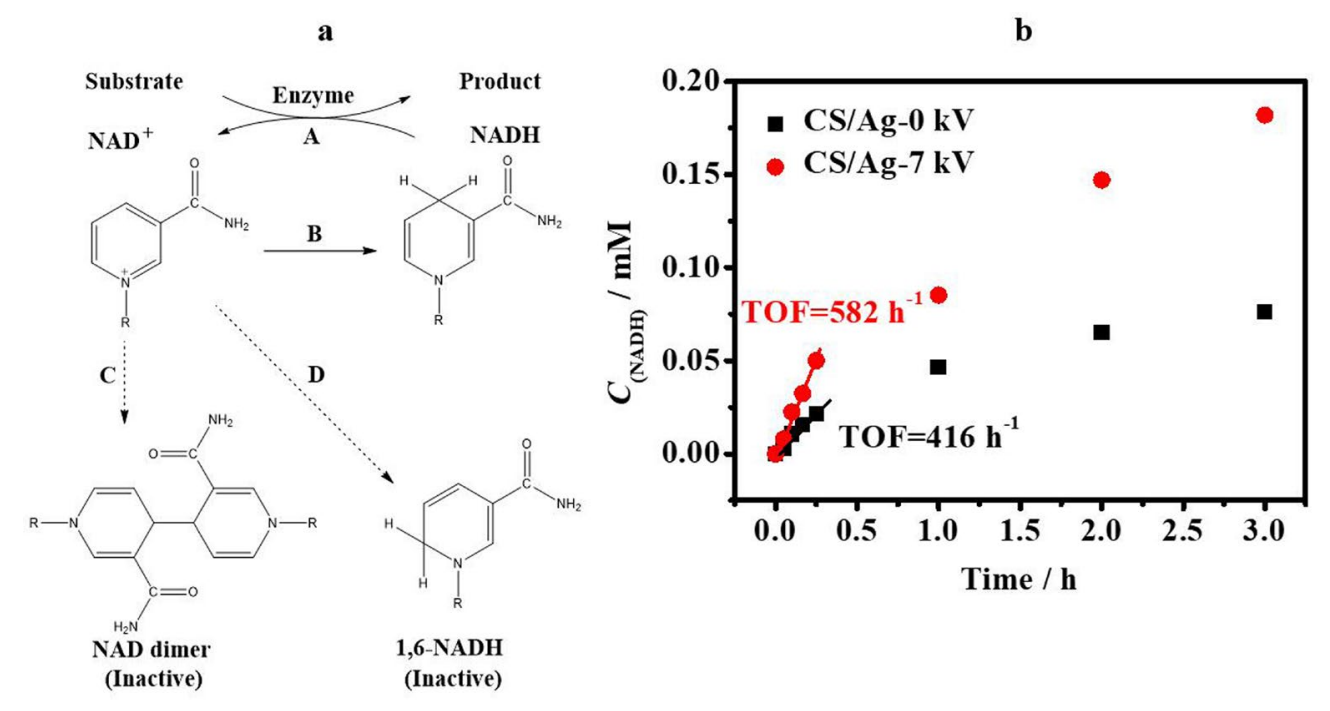

Figure 6. Catalytic performance of $\mathrm{CS} / \mathrm{Ag}$ nanocomposites in NADH regeneration: a) reaction pathways and $\mathrm{b}$ ) variation of NADH concentration as a function of time. Reaction conditions are available in the Methods.

The catalytic performance of the homogeneous Ag NPs was assessed in the bio-cofactor $\mathrm{NADH}$ regeneration as a representative reaction. In addition to high activity, selectivity is even more crucial and challenging in the 21 century for chemical processes with complicated reaction networks. ${ }^{36} \mathrm{NADH}$ is a critical reducing agent (hydride donor) participating in many enzymatic reductions for the synthesis of pharmaceutical/chemical products (path A in Figure 6a) and its high cost $(\$ 3,000 / \mathrm{mol})$ and stoichiometric use make the regeneration (path B in Figure 6a) essential for practical applications. ${ }^{37-40}$ The regeneration process is challenging due to side reactions leading to the inactive dimer and 1,6-NADH isomer (paths $\mathrm{C}$ and D in Figure 6a) whereas the enzymatically active $(1,4-) \mathrm{NADH}$ is the target. ${ }^{38}$ As shown it is encouraging to observe (Figure 6b) that both $\mathrm{CS} / \mathrm{Ag}$ nanocomposites are active in this reaction and exhibit $T O F$ s of up to $582 \mathrm{~h}^{-1}\left(\mathrm{pH}=7,37^{\circ} \mathrm{C}, 10 \mathrm{~atm} \mathrm{H}_{2}\right)$, an order of magnitude higher than our recent reported data $\left(55 \mathrm{~h}^{-1}\right)$ obtained over supported $\mathrm{Pt} \mathrm{NPs}\left(\mathrm{pH}=7,37^{\circ} \mathrm{C}, 9\right.$ atm $\left.\mathrm{H}_{2}\right)^{39}$ and 80 times greater than that $\left(\sim 7 \mathrm{~h}^{-1}\right)$ catalyzed by homogeneous $\mathrm{Ir}$ complex $(\mathrm{pH}=$ $\left.7,25^{\circ} \mathrm{C}, 1 \mathrm{~atm} \mathrm{H}_{2}\right)^{40}$. Of significance, the regeneration procedure exclusively produced the 
enzymatically active $\mathrm{NADH}$, i.e. $100 \%$ selectivity, as confirmed by an independent enzymatic assay (see Methods).

The synthesized CS/Ag nanocomposites as heterogeneous films were examined by the bactericidal activity against $S$. aureus (see Figure S8). Incorporation of $\mathrm{Ag}^{0}$ can increase the mean zone of inhibition owing to the super antimicrobial qualities of $\mathrm{Ag} \mathrm{NPs}\left(\mathrm{Ag}^{0}\right.$ at $3.4 \pm 0.7$ $\mathrm{nm}$ ), in line with the literature where smaller Ag NPs exhibited better antibacterial performance. $^{41,42}$

To demonstrate the wider applicability of this method for the synthesis of metal NPs, we have extended the work to other metals $($ i.e. $\mathrm{Cu}, \mathrm{Au}$, and $\mathrm{Pt}$, precursor valencies ranging from +2 to +4$)$ to suit the demand in different potential applications based on metal NPs $\left(e . g . \mathrm{H}^{+} / \mathrm{H}_{2}\right.$ reactions, electron mediator, water treatment, catalytic oxidation, coupling reactions, and carboxylation, etc $\left.{ }^{43-49}\right)$. The synthesized metal NPs (Figure 7a-c), were all nanoscale $(d=2.0$ $(\mathrm{Cu}), 1.4(\mathrm{Au})$, and $2.1(\mathrm{Pt}) \mathrm{nm})$ with narrow size distributions, illustrating effective control of metal NPs. XPS analysis (Figure 7d-f) confirmed the formation of metallic $\mathrm{Cu}(932.3 \mathrm{eV})$, $\mathrm{Au}(83.5 \mathrm{eV})$, and $\mathrm{Pt}(70.8 \mathrm{eV})$, showing the reductive efficiency of the reported method. The $\mathrm{Cu}$ metallic state is further evidenced by the Auger $\mathrm{Cu}$ LMM plot where only one peak at $567.9 \mathrm{eV}$ (for $\left.\mathrm{Cu}^{0}\right)^{44,45}$ was present (see Figure 7d inset). It should be mentioned that at a high Pt loading (6 wt\%), the precursor $\mathrm{Pt}^{4+}$ was only reduced to $\mathrm{Pt}^{2+}$ (see Figure $\mathbf{S 9}, \mathrm{Pt}^{2+}$ at 72.0 $\mathrm{eV}$ ). Decreasing the loading to $1 \mathrm{wt} \%$ led to complete reduction of $\mathrm{Pt}^{4+}$ to $\mathrm{Pt}^{0}$ as shown in Figure 7f. The sizes of metal NPs prepared using this electric-field-driven phase inversion method are, in general, smaller than the recently reported extreme cathode corrosion approach where the Au and Pt NPs produced were between 3-30 nm and Rh was $\sim 4 \mathrm{~nm} .{ }^{5,7}$ 


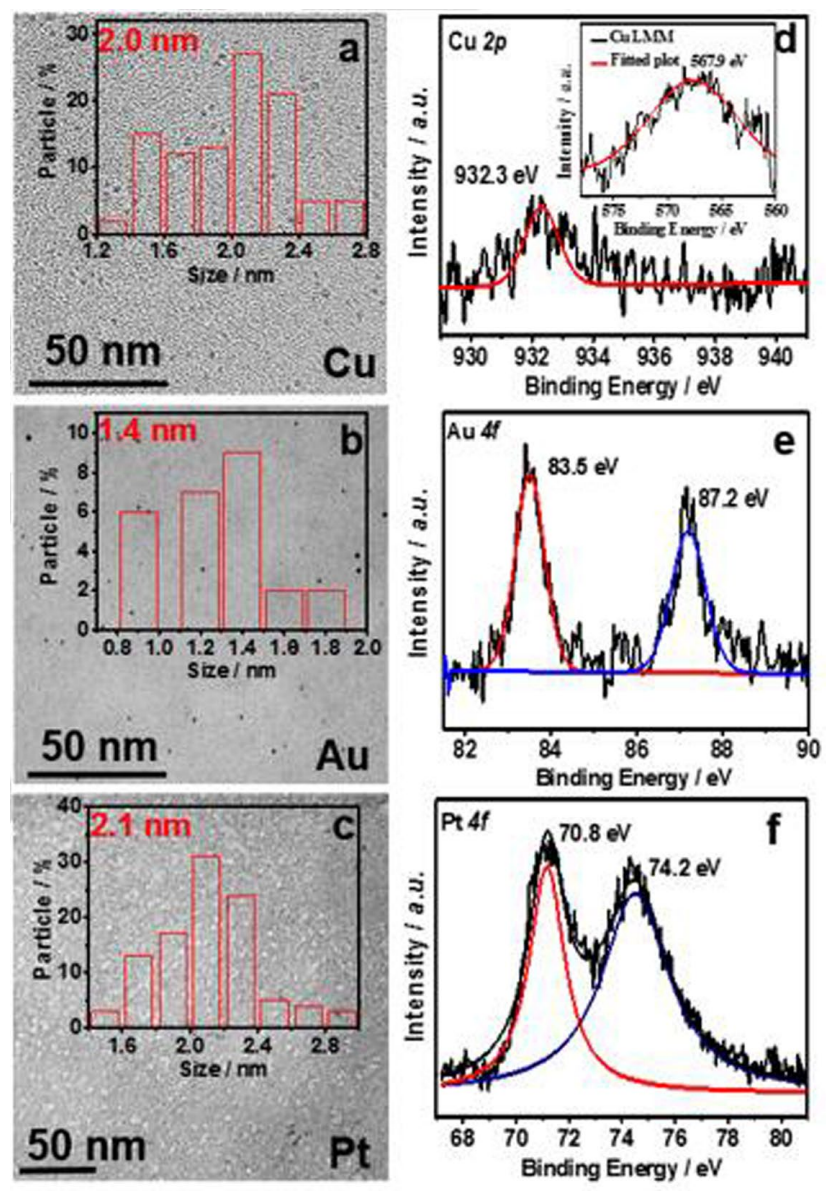

Figure 7. TEM images for a) $\mathrm{CS} / \mathrm{Cu}, \mathrm{b}) \mathrm{CS} / \mathrm{Au}$ and c) $\mathrm{CS} / \mathrm{Pt}(1 \mathrm{wt} \%$, loadings for $\mathrm{a}, \mathrm{b}$ are 6 wt\%) nanocomposites; inset: metal particle size distribution. Corresponding XPS spectra of $\mathrm{Cu} 2 p, \mathrm{Au} 4 f$ and $\mathrm{Pt} 4 f$ are shown in d-f; inset of d: Auger spectra of the Cu LMM for the $\mathrm{CS} / \mathrm{Cu}$ nanocomposite.

\section{Conclusions}

In summary, it has been shown that the electric-field-driven phase inversion is a facile, efficient and clean method for synthesizing narrow-distributed and water-soluble metal nanoparticles. Bioderived chitosan can stabilize the metal nanoparticles and formic acid acts as both a solvent (for the polymer) and a reducing agent (for the metal). The electric field applied can modify the hydrogen bonding environment of the chitosan and promote the stabilization and reduction efficiency of metal nanoparticles. The produced chitosan/Ag nanocomposites can be directly used in homogenous (water-soluble) form for catalysis and heterogeneous form (as a film) for antibacterial application. The method has been successfully 
extended to other metals (up to +4 valency) including $\mathrm{Cu}, \mathrm{Au}$ and $\mathrm{Pt}$ as examples and obtain particles of $\sim 1-2 \mathrm{~nm}$ mean sizes, showing the universality and efficiency. Looking forward, it is anticipated that the use of electric-field-driven phase inversion will also prove valuable when synthesizing other fundamental nanomaterials for catalysis and beyond, further expanding the range of potential applications.

\section{ASSOCIATED CONTENT}

\section{Supporting Information}

The Supporting Information is available free of charge on the ACS Publications website at DOI:

XPS spectrum of the nanocomposites; Fitting curves and the fitted results of the XPS O1s, N1s and C1s curve; Water-solubility of the nanocomposites; The UV-vis spectra and FTIR of $\mathrm{CS}$ and CS/Ag nanocomposites immersed in distilled water; TEM images for CS/Pt (6 wt $\%$ ) and corresponding XPS spectrum of $\mathrm{Pt} 4 \mathrm{f}$.

\section{AUTHOR INFORMATION}

Corresponding Author

*E-mail: zhouqi@,nbut.edu.cn, x.wang@,abdn.ac.uk

\section{Notes}

The authors declare no competing financial interest.

\section{ACKNOWLEDGMENTS}

Funding from the National Natural Science Foundation of China (No. 51203081), Natural Science Foundation of Zhejiang Province (LY16E030005), Opening Foundation from Zhejiang Provincial Key Laboratory of Advanced Chemical Engineering Manufacture Technology (ACEMT-17-01), and K.C. Wong Magna Fund of Ningbo University is 
gratefully acknowledged. X.W. also acknowledges financial support from The Royal Society (RG150001 and IE150611).

\section{REFERENCES}

1 Lei, Y.; Mehmood, F.; Lee, S.; Greeley, J.; Lee, B.; Seifert, S.; Winans, R. E.; Elam, J. W.; Meyer, R. J.; Redfern, P. C.; Teschner, D.; Schlögl, R.; Pellin, M. J.; Curtiss L. A.; Vajda, S. Increased silver activity for direct propylene epoxidation via subnanometer size effects, Science, 2010, 328, 224-228.

2 Scholl, J. A.; Koh, A. L.; Dionne, J. A. Quantum plasmon resonances of individual metallic nanoparticles. Nature, 2012, 483, 421-468.

3 Chhowalla, M.; Shin, H. S.; Eda, G.; Li, L. J.; Loh K. P.; Zhang, H. The chemistry of two-dimensional layered transition metal dichalcogenide nanosheets. Nat. Chem., 2013, 5, 263-275.

4 Munnik, P.; Velthoen, M. E. Z.; de Jongh, P. E.; de Jong K. P.; Gommes, C. J. Nanoparticle growth in supported nickel catalysts during methanation reaction-larger is better. Angew. Chem. Int. Ed., 2014, 53, 9493-9497.

5 Rodriguez, P.; Tichelaar, F. D.; Koper M. T. M.; Yanson, A. I. Cathodic corrosion as a facile and effective method to prepare clean metal alloy nanoparticles. J. Am. Chem. Soc., 2011, 133, 17626-17629.

6 Tavakkoli, M.; Kallio, T.; Reynaud, O.; Nasibulin, A. G.; Johans, C.; Sainio, J.; Jiang, H.; Kauppinen E. I.; Laasonen, K. Single-shell carbon-encapsulated iron nanoparticles: synthesis and high electrocatalytic activity for hydrogen evolution reaction. Angew. Chem. Int. Ed., 2015, 54, 4535-4538.

7 Yanson, A. I.; Rodriguez, P.; Garcia-Araez, N.; Mom, R. V.; Tichelaar, F. D.; Koper, M. T. M. Cathodic corrosion: a quick, clean, and versatile method for the synthesis of metallic nanoparticles. Angew. Chem. Int. Ed., 2011, 50, 6346-6350. 
8 Dong, X.; Ji, X.; Wu, H.; Zhao, L.; Li J.; Yang, W. Shape control of silver nanoparticles by stepwise citrate reduction. J. Phys. Chem. C, 2009, 113, 6573-6576.

9 Wang, L.; Li, H.; Tian J.; Sun, X. Monodisperse, micrometer-scale, highly crystalline, nanotextured Ag dendrites: rapid, large-scale, wet-chemical synthesis and their application as SERS substrates. ACS Appl. Mater. Interfaces, 2010, 2, 2987-2991.

10 Zhu Q.L.; Xu, Q. Immobilization of ultrafine metal nanoparticles to high-surface-area materials and their catalytic applications. Chem, 2016, 1, 220-245.

11 Bae, K. H.; Park, M.; Do, M. J.; Lee, N.; Ryu, J. H.; Kim, G. W.; Kim, C.; Park, T. G.; Hyeon, T. Chitosan oligosaccharide-stabilized ferrimagnetic iron oxide nanocubes for magnetically modulated cancer hyperthermia. ACS Nano, 2012, 6, 5266-5273.

12 Jung, J. H.; Lee, J. H.; Shinkai, S. Functionalized magnetic nanoparticles as chemosensors and adsorbents for toxic metal ions in environmental and biological fields. Chem. Soc. Rev., 2011, 40, 4464-4474.

13 Virkutyte, J.; Varma, R. S. Green synthesis of metal nanoparticles: biodegradable polymers and enzymes in stabilization and surface functionalization. Chem. Sci., 2011, $2,837-846$.

14 Wang, X.; Perret, N.; Delannoy, L.; Louis, C.; Keane, M. A. Selective gas phase hydrogenation of nitroarenes over $\mathrm{Mo}_{2} \mathrm{C}$-supported Au-Pd. Catal. Sci. Technol., 2016, 6, 6932-6941.

15 Zhang, X.; Chen, L.; Yun, J.; Wang X.; Kong, J. Constructing magnetic Si-C-Fe hybrid microspheres for room temperature nitroarenes reduction. J. Mater. Chem. A, 2017, 5, 10986-10997.

16 Morrow, B. H.; Payne G. F.; Shen, J. pH-responsive self-assembly of polysaccharide through a rugged energy landscape. J. Am. Chem. Soc., 2015, 137, 13024-13030. 
17 Nhung, T. T.; Lee, S.-W. Green synthesis of asymmetrically textured silver mesoflowers (AgMFs) as highly sensitive SERS substrates. ACS Appl. Mater. Interfaces, 2014, 6, 21335-21345.

18 Pigaleva, M. A.; Portnov, I. V.; Rudov, A. A.; Blagodatskikh, I. V.; Grigoriev, T. E. Gallyamov, M. O.; Potemkin, I. I. Stabilization of chitosan aggregates at the nanoscale in solutions in carbonic acid. Macromolecules, 2014, 47, 5749-5758.

19 Yui, T.; Imada, K.; Okuyama, K.; Obata, Y.; Suzuki, K.; Ogawa, K. Molecular and crystal structure of the anhydrous form of chitosan. Macromolecules, 1994, 27, 76017605.

20 An, B.; Zhang, J.; Cheng, K.; Ji, P.; Wang, C.; Lin, W. Confinement of ultrasmall $\mathrm{Cu} / \mathrm{ZnOx}$ nanoparticles in metal-organic frameworks for selective methanol synthesis from catalytic hydrogenation of $\mathrm{CO}_{2}$. J. Am. Chem. Soc., 2017, 139, 3834-3840.

21 Deng, D.; Yu, L.; Chen, X.; Wang, G.; Jin, L.; Pan, X.; Deng, J.; Sun G.; X. Bao, Iron encapsulated within pod-like carbon nanotubes for oxygen reduction reaction. Angew. Chem. Int. Ed., 2013, 52, 371-375.

22 Frindy, S.; el Kadib, A.; Lahcini, M.; Primo A.; García, H. Copper Nanoparticles Stabilized in a Porous Chitosan Aerogel as a Heterogeneous Catalyst for C-S Crosscoupling. ChemCatChem, 2015, 7, 3307-3315.

23 Guibal, E. Heterogeneous catalysis on chitosan-based materials: a review. Prog. Polym. Sci., 2005, 30, 71-109.

24 Sahoo, B.; Surkus, A.-E.; Pohl, M.-M.; Radnik, J.; Schneider, M.; Bachmann, S.; Scalone, M.; Junge K.; Beller, M. A biomass-derived non-noble cobalt catalyst for selective hydrodehalogenation of alkyl and (hetero) aryl halides. Angew. Chem. Int. Ed., 2017, 56, 11242-11247. 
25 Zhou, Q.; Cong, Y.; Wu, N.; Loo, L. S. The microstructure of polyamide 6 and polyamide 6/polyhedral oligomeric silsesquioxane nanocomposites synthesized by phase inversion procedure under electric field. Appl. Surf. Sci., 2015, 357, 1454-1462.

26 Zhou, Q.; Zhang, J.; Wang, Y.; Wang, W.; Yao, S.; Cong, Y.; Fang, J. Synergistic effects of filler-migration and moisture on the surface structure of polyamide 6 composites under an electric field. $R S C A d v .$, 2016, 6, 95535-95541.

27 Begum, S. N. S.; Aswal, V. K.; Ramasamy, R. P. Small-angle neutron scattering and spectroscopic investigations of Ag fractal formation in chitosan-Ag nanocomposite facilitated by hydrazine hydrate. J. Phys. Chem. C, 2016, 120, 2400-2410.

28 De Silva, R. T.; Mantilaka, M. G. P.; Ratnayake, S. P.; Amaratunga, G. A. J.; de Silva, K. M. N. Nano-MgO reinforced chitosan nanocomposites for high performance packaging applications with improved mechanical, thermal and barrier properties. Carbohyd. Polym., 2017, 157, 739-747.

29 Wang, Z.; Xu, C.; Zhao, M.; Zhao, C. One-pot synthesis of narrowly distributed silver nanoparticles using phenolic-hydroxyl modified chitosan and their antimicrobial activity. $R S C A d v .$, 2014, 4, 47021-47030.

30 Liedel, C.; Pester, C. W.; Ruppel, M.; Lewin, C.; Pavan, M. J.; Urban, V. S.; Shenhar, R.; Bösecke, P.; Böker, A. Block copolymer nanocomposites in electric fields: kinetics of alignment. ACS Macro Lett., 2012, 2, 53-58.

31 Liedel, C.; Schindler, K. A.; Pavan, M. J.; Lewin, C.; Pester, C. W.; Ruppel, M.; Urban, V. S.; Shenhar, R.; Böker, A. Electric-field-induced alignment of block copolymer/nanoparticle blends. Small, 2013, 9, 3276-3281.

32 Mady, A. H.; Baynosa, M. L.; Tuma, D.; Shim, J.J. Facile microwave-assisted green synthesis of $\mathrm{Ag}-\mathrm{ZnFe}_{2} \mathrm{O}_{4} @ \mathrm{rGO}$ nanocomposites for efficient removal of organic dyes under UV-and visible-light irradiation. Appl. Catal. B: Environ., 2017, 203, 416-427. 
33 Rogach, A. L.; Shevchenko, G. P.; Afanas'ev, Z. M.; Sviridov, V. V. Changes in the morphology and optical absorption of colloidal silver reduced with formic acid in the polymer matrix under UV irradiation. J. Phys. Chem. B, 1997, 101, 8129-8132.

34 Kang, J.; Liu, H.; Zheng, Y.M.; Qu, J.; Chen, J. P. Systematic study of synergistic and antagonistic effects on adsorption of tetracycline and copper onto a chitosan. $J$. Colloid Interf. Sci, 2010, 344, 117-125.

35 Son, W. K.; Youk J. H.; Park, W. H. Antimicrobial cellulose acetate nanofibers containing silver nanoparticles. Carbohyd. Polym., 2006, 65, 430-434.

36 Somorjai, G.; Kliewer, C.Reaction selectivity in heterogeneous catalysis. React. Kinet. Catal. Lett., 2009, 96, 191-208.

37 Bornscheuer, U. T.; Huisman, G. W.; Kazlauskas, R. J.; Lutz, S.; Moore, J. C.; Robins, K. Engineering the third wave of biocatalysis. Nature, 2012, 485, 185-194.

38 Wang, X.; Saba, T.; Yiu, H. H. P.; Howe, R. F.; Anderson, J. A.; Shi, J. Cofactor NAD(P)H regeneration inspired by heterogeneous pathways. Chem, 2017, 2, 621-654.

39 Wang, X.; Yiu, H.H.P. Heterogeneous catalysis mediated cofactor NADH regeneration for enzymatic reduction. ACS Catal., 2016, 6, 1880-1886.

40 Maenaka, Y.; Suenobu T.; Fukuzumi, S. Efficient catalytic interconversion between $\mathrm{NADH}$ and $\mathrm{NAD}^{+}$accompanied by generation and consumption of hydrogen with a water-soluble iridium complex at ambient pressure and temperature. J. Am. Chem. Soc., 2012, 134, 367-374.

41 Archana, D.; Singh, B. K.; Dutta, J.; Dutta, P. K. Chitosan-PVP-nano silver oxide wound dressing: in vitro and in vivo evaluation. Int. J. Biol. Macromol., 2015, 73, 4957.

42 Gabriel, J. S.; Gonzaga, V. A. M.; Poli, A. L.; Schmitt, C. C. Photochemical synthesis of silver nanoparticles on chitosans/montmorillonite nanocomposite films and antibacterial activity. Carbohyd. Polym., 2017, 171, 202-210. 
43 Song, H.-K.; Lee, S. H.; Won, K.; Park, J. H.; Kim, J. K.; Lee, H.; Moon, S.-J.; Kim, D. K.; Park, C. B. Electrochemical regeneration of NADH enhanced by platinum nanoparticles. Angew. Chem. Int. Ed., 2008, 47, 1749-1752.

44 Liu, P.; Hensen, E. J. M. Highly efficient and robust $\mathrm{Au} / \mathrm{MgCuCr}_{2} \mathrm{O}_{4}$ catalyst for gasphase oxidation of ethanol to acetaldehyde. J. Am. Chem. Soc., 2013, 135, 1403214035.

45 Paracchino, A.; Laporte, V.; Sivula, K.; Gratzel, M.; Thimsen, E. Highly active oxide photocathode for photoelectrochemical water reduction. Nat. Mater., 2011, 10, 456461.

46 Shen, C.; Xu, J.; Yu, W.; Zhang, P. A highly active and easily recoverable chitosan@ copper catalyst for the $\mathrm{C}-\mathrm{S}$ coupling and its application in the synthesis of zolimidine. Green Chem., 2014, 16, 3007-3012.

47 Gross, E.; Shu, X.Z.; Alayoglu, S.; Bechtel, H. A.; Martin, M. C.; Toste, F. D.; Somorjai, G. A. In situ IR and X-ray high spatial-resolution microspectroscopy measurements of multistep organic transformation in flow microreactor catalyzed by Au nanoclusters. J. Am. Chem. Soc., 2014, 136, 3624-3629.

48 Lai, S. C. S.; Dudin, P. V.; Macpherson, J. V.; Unwin, P. R. Visualizing zeptomole (electro) catalysis at single nanoparticles within an ensemble. J. Am. Chem. Soc., 2011, $133,10744-10747$.

49 Pritchard, J.; Filonenko, G. A.; van Putten, R.; Hensen E. J. M.; Pidko, E. A. Heterogeneous and homogeneous catalysis for the hydrogenation of carboxylic acid derivatives: history, advances and future directions. Chem. Soc. Rev., 2015, 44, 38083833. 